\title{
Applications of Mössbauer Spectroscopy to Studies of Quasicrystals
}

\author{
Z.M. STADNIK \\ Department of Physics, University of Ottawa \\ Ottawa, Ontario K1N 6N5, Canada
}

\begin{abstract}
A review is presented of the application of the Mössbauer spectroscopy to the study of quasicrystals. The usefulness of the Mössbauer spectroscopy to solving the atomic structure of a complex icosahedral system is illustrated. The role of the Mössbauer spectroscopy in studies of magnetism of quasicrystals is discussed. The Mössbauer spectroscopy results on phason dynamics in quasicrystals are reviewed.
\end{abstract}

PACS numbers: 75.50.Kj, 75.50.Lk, 76.80.+y

\section{Introduction}

Solids are traditionally divided into two groups: crystalline and amorphous. The dramatic discovery of an icosahedral Al-Mn alloy by Shechtman et al. [1] extended this dichotomous division by introducing the notion of quasicrystals (QCs). These are compounds that possess a new type of long-range translational order, quasiperiodicity, and a non-crystallographic orientational order associated with the classically forbidden fivefold, eightfold, tenfold, and twelvefold symmetry axes [2]. A central problem in condensed matter physics is determining whether quasiperiodicity leads to physical properties which are significantly different from those of crystalline and amorphous materials.

The Mössbauer spectroscopy (MS) is a local probe which has been used in studies of QCs [3] to obtain three types of information. First, local atomic structure has been studied through the electric quadrupole interactions. The electric quadrupole interaction parameters have been calculated for possible structural models of a given QC and compared with those derived from the Mössbauer spectra. This has led to the first solution of the structure of the ternary $\mathrm{Al}-\mathrm{Cu}-\mathrm{Fe}$ icosahedral QC. Second, magnetic properties of QCs have been studied through the magnetic dipole interaction. Third, phason dynamics, which is the lattice dynamics specific to QCs, has been investigated via MS high-temperature measurements. 


\section{Applications}

\subsection{Atomic structure}

In spite of enormous experimental and theoretical effort, the atomic structures of QCs, except for the icosahedral binary $\mathrm{QC} \mathrm{YbCd}_{5.7}$ [4], have not been solved yet. A structural model for an icosahedral $\mathrm{Al}-\mathrm{Cu}-\mathrm{Fe} \mathrm{QC}$ was devised by Cockayne et al. [5]. It is in the form of a $1 / 1$ approximant [2] with 128 atoms per unit cell. The model is based on synchrotron radiation X-ray diffraction data from a single-grain $\mathrm{Al}-\mathrm{Cu}-\mathrm{Fe} \mathrm{QC}$. For this original model, and its two versions: the relaxed model and the modified model, the electric field gradient (EFG) calculations at the $\mathrm{Fe}$ and $\mathrm{Al}$ sites were carried out [6]. The relaxed model is the original model with an ab initio relaxation of atomic positions, whereas in the modified model the positions of $\mathrm{Cu}$ and $\mathrm{Fe}$ sites were interchanged [6]. The basic features of the modified model are shown in Fig. 1.
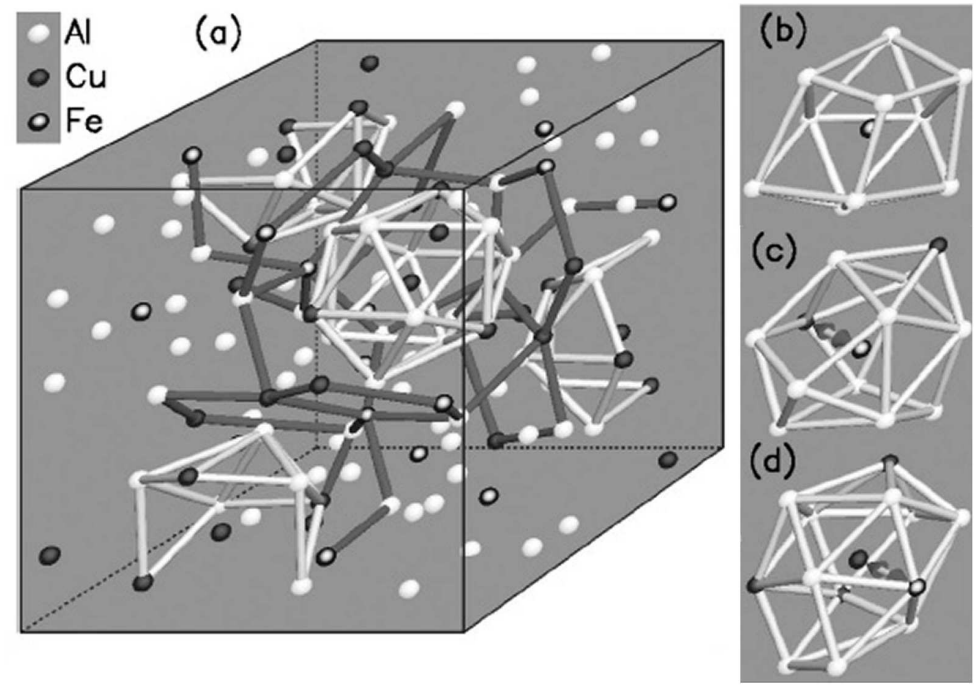

Fig. 1. (a) Unit cell of the modified Cockayne model. Four distorted icosahedra (light bonds) surround the $\mathrm{Cu} 3$ atoms and four pentagonal dodecahedra (dark bonds) surround the icosahedra. The subfigures show the nearest-neighbor environment of (b) Fe0, (c) Fe1, and (d) Cu1 atom. Double arrows indicate Fe1 and Cu1 atoms that have been interchanged [6].

A direct comparison between the ${ }^{57} \mathrm{Fe}$ Mössbauer spectrum of the icosahedral $\mathrm{Al}_{62.5} \mathrm{Cu}_{24.5} \mathrm{Fe}_{13} \mathrm{QC}$ at $4.3 \mathrm{~K}$ measured in zero external magnetic field and the theoretical spectra generated for the EFG parameters calculated for the original, relaxed, and modified Cockayne models is shown in Fig. 2. It is clear that the spectra generated for the original (Fig. 2a) and the relaxed Cockayne model (Fig. 2b) cannot even account for the shape of the experimental spectrum. The 

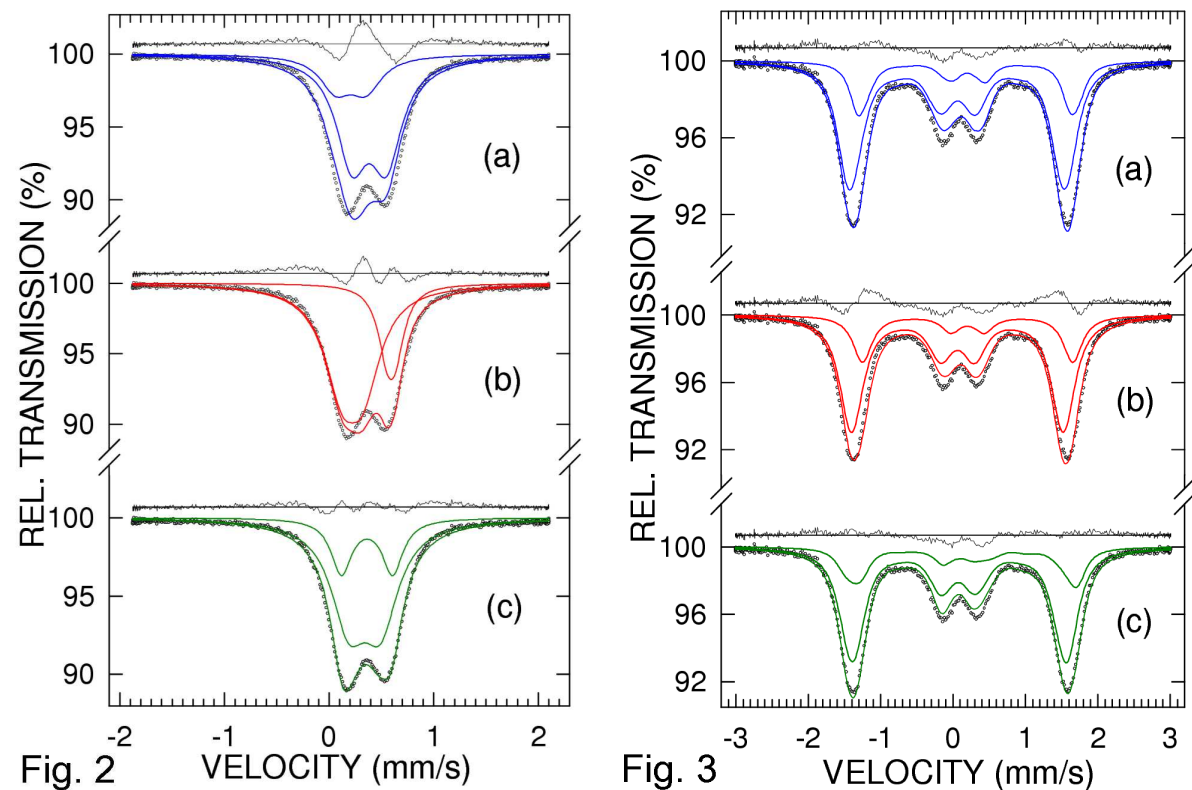

Fig. 2. Comparison of the ${ }^{75} \mathrm{Fe}$ Mössbauer spectrum of icosahedral $\mathrm{Al}_{62.5} \mathrm{Cu}_{24.5} \mathrm{Fe}_{13}$ at $4.3 \mathrm{~K}$ (open circles) with the theoretical spectra (solid lines) generated for the values of the EFG parameters calculated for the (a) original, (b) relaxed, and (c) modified Cockayne models. The zero-velocity origin is relative to $\alpha$-Fe at room temperature. The differences between the experimental and theoretical spectra are shown above each spectrum [6].

Fig. 3. Comparison of the ${ }^{75} \mathrm{Fe}$ Mössbauer spectrum of icosahedral $\mathrm{Al}_{62.5} \mathrm{Cu}_{24.5} \mathrm{Fe}_{13}$ at $4.4 \mathrm{~K}$ measured in an external magnetic field of 9.0 $\mathrm{T}$ (open circles) with the theoretical spectra (solid lines) generated for the values of the EFG parameters calculated for the (a) original, (b) relaxed, and (c) modified Cockayne models. The zero-velocity origin is relative to the ${ }^{57} \mathrm{Co}(\mathrm{Rh})$ source at $4.4 \mathrm{~K}$. The differences between the experimental and theoretical spectra are shown above each spectrum [6].

Mössbauer spectrum generated for the modified Cockayne model (Fig. 2c) describes the experimental spectrum well. A similar comparison between the ${ }^{75} \mathrm{Fe}$ Mössbauer spectrum of the icosahedral $\mathrm{Al}_{62.5} \mathrm{Cu}_{24.5} \mathrm{Fe}_{13} \mathrm{QC}$ at $4.4 \mathrm{~K}$ measured in an external magnetic field of $9.0 \mathrm{~T}$ and the theoretical spectra generated for the EFG parameters calculated for the original, relaxed, and modified Cockayne models (Fig. 3) shows that the spectrum generated for the modified Cockayne model fit best the experimental in-field spectrum. This example demonstrates that the combination of theoretical EFG calculations for a possible structural model of a complex alloy with the MS data can be used to solve the atomic structure of that compound. 

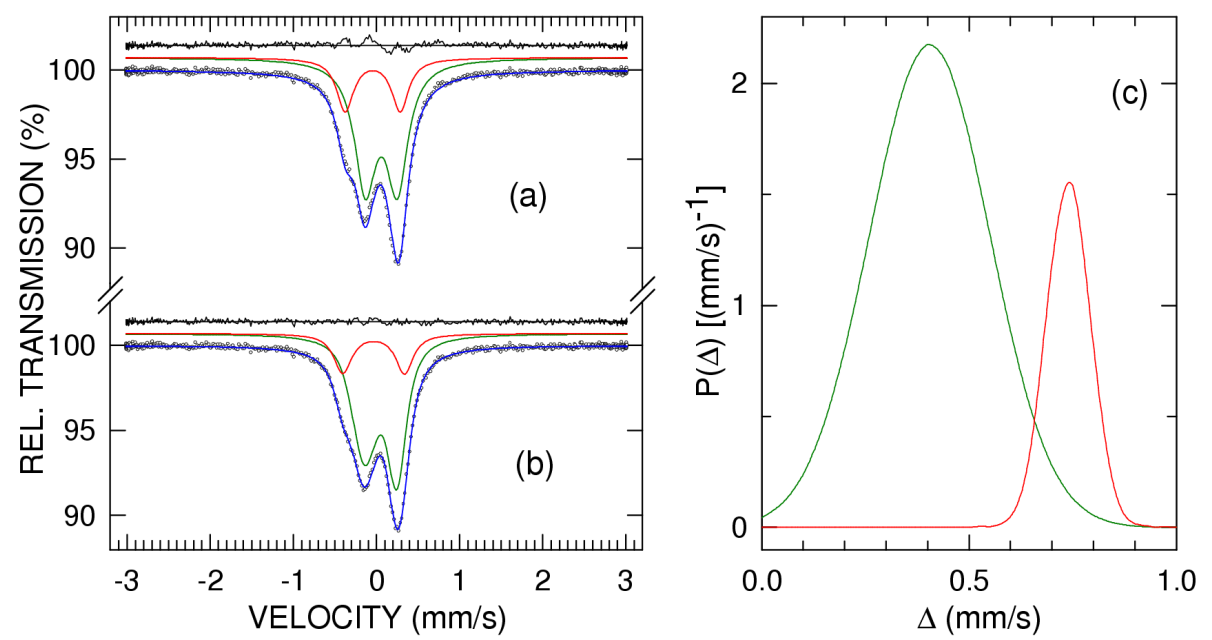

Fig. 4. The ${ }^{75} \mathrm{Fe}$ Mössbauer spectrum of the decagonal $\mathrm{Al}_{70} \mathrm{Co}_{15} \mathrm{Ni}_{14.9} \mathrm{Fe}_{0.1}$ at $5.3 \mathrm{~K}$ fitted (solid line) (a) with two symmetric quadrupole doublets and (b) with two $P(\Delta)$ components shown in (c). The zero-velocity origin in (a) and (b) is relative to the ${ }^{57} \mathrm{Co}(\mathrm{Rh})$ source at $5.3 \mathrm{~K}$. The differences between the experimental and fitted spectra are shown above each spectrum [7].

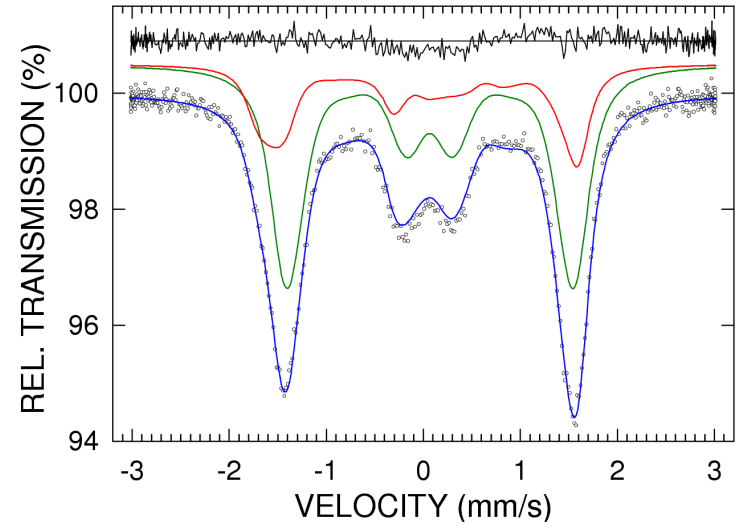

Fig. 5. The ${ }^{75} \mathrm{Fe}$ Mössbauer spectrum of the decagonal $\mathrm{Al}_{70} \mathrm{Co}_{15} \mathrm{Ni}_{14.9} \mathrm{Fe}_{0.1}$ at $5.4 \mathrm{~K}$ in an external magnetic field of $9.0 \mathrm{~T}$ fitted (solid line) with two components. The zero-velocity origin is relative to the ${ }^{57} \mathrm{Co}(\mathrm{Rh})$ source at $5.4 \mathrm{~K}$. The difference between the experimental and fitted spectra is shown above the spectrum [7].

${ }^{75} \mathrm{Fe}$ Mössbauer zero-field spectra of most nonmagnetic QCs consist of two broad and structureless lines [3] which result from the multiplicity of Fe sites in a quasicrystalline structure. The notable exceptions are the spectra of Al-Co-based decagonal QCs which clearly show a distinct structure (Fig. 4). A successful fit of 
the zero-field Mössbauer spectrum of the decagonal $\mathrm{Al}_{70} \mathrm{Co}_{15} \mathrm{Ni}_{14.9} \mathrm{Fe}_{0.1}$ QC with two distinct distributions of the quadrupole splittings $P(\Delta)$ (Fig. $4 \mathrm{~b}$ and c) provides evidence for the existence of two classes of iron sites in this QC. The fit of the Mössbauer spectrum of the decagonal $\mathrm{Al}_{70} \mathrm{Co}_{15} \mathrm{Ni}_{14.9} \mathrm{Fe}_{0.1} \mathrm{QC}$ measured in an external magnetic field of $9.0 \mathrm{~T}$ (Fig. 5) provided a complete set of the EFG parameters [7] characterizing the iron sites in this QC. Comparing these parameters with those obtained from $a b$ initio EFG calculations for possible structural models of the decagonal $\mathrm{Al}-\mathrm{Co}-\mathrm{Ni}$ QC [8] could lead to the solution of the atomic structure of this QC.

\subsection{Magnetism of quasicrystals}

One of the central issues in the physics of QCs is that of the possibility of long-range magnetic order. Initial intuition suggests that quasiperiodicity necessarily leads to geometric frustration and is therefore incompatible with longrange magnetic order. However, many theoretical models dealing with magnetism in QCs indicate the possibility of long-range order in these alloys. On the experimental side, all known QCs are either diamagnets, paramagnets, or spin-glasses [3]. The claim [9] of the existence of long-range magnetic order in icosahedral $\mathrm{RE}-\mathrm{Mg}-\mathrm{Zn}(\mathrm{RE}=$ rare earth) QCs was shown [10] to result from the presence of magnetic impurities in the studied samples.
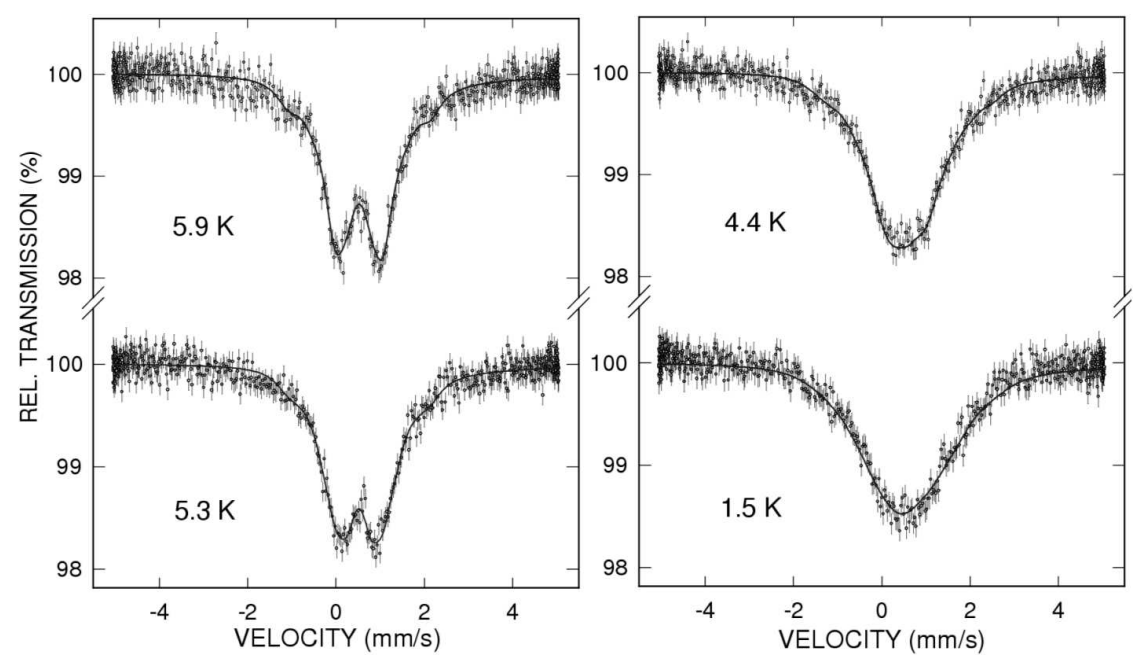

Fig. 6. ${ }^{155} \mathrm{Gd}$ Mössbauer spectra of the icosahedral $\mathrm{Ag}_{50} \operatorname{In}_{36} \mathrm{Gd}_{14}$ at 5.9, 5.3, 4.4 and $1.5 \mathrm{~K}$. Solid lines are fits, as described in the text. The zero-velocity origin is relative to the ${ }^{155} \mathrm{Eu}\left(\mathrm{SmPd}_{3}\right)$ source [12].

Recently, new metastable icosahedral QCs were discovered in the $\mathrm{Ag}_{50} \mathrm{In}_{36} \mathrm{RE}_{14}$ system [11]. These new QCs are expected to possess well localized and sizeable $4 f$ magnetic moments on the $\mathrm{R}$ elements and perhaps exhibit long- 


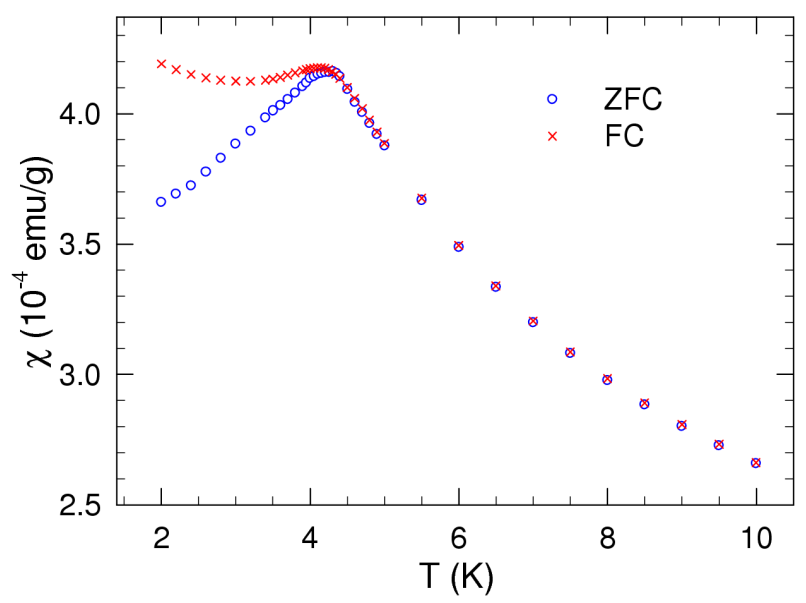

Fig. 7. The temperature dependence of the zero-field-cooled (ZFC) and field-cooled (FC) magnetic susceptibility of the icosahedral $\mathrm{Ag}_{50} \operatorname{In}_{36} \mathrm{Gd}_{14}$, measured in an external magnetic field of 30 Oe [12].

-range magnetic order. One of these QCs, $\mathrm{Ag}_{50} \mathrm{In}_{36} \mathrm{Gd}_{14}$, has been studied with ${ }^{155} \mathrm{Gd}$ MS and magnetization measurements [12]. Its selected low-temperature ${ }^{155} \mathrm{Gd}$ Mössbauer spectra are shown in Fig. 6 . The spectra at 5.9 and $5.3 \mathrm{~K}$ were fitted with a distribution of quadrupole splittings [12]. Those at 4.4 and $1.5 \mathrm{~K}$ (Fig. 6) show the presence of the combined electric quadrupole and magnetic dipole interactions. The presence of the magnetic dipole interaction in the spectrum at $4.4 \mathrm{~K}$, and its absence in the spectrum at $5.3 \mathrm{~K}$, shows that magnetic ordering sets in at a temperature between 4.4 and $5.3 \mathrm{~K}$. The nature of this magnetic ordering is revealed in the temperature dependence of the magnetic susceptibility (Fig. 7). The occurrence of a bifurcation between the ZFC and FC data at 4.25(5) K proves that the icosahedral $\mathrm{Ag}_{50} \mathrm{In}_{36} \mathrm{Gd}_{14} \mathrm{QC}$ does not have long-range magnetic order but is a spin glass.

\subsection{Phason dynamics}

The elementary dynamic excitations in QCs are conventional phonons and, specific to a quasicrystalline structure, phasons [2]. Phasons involve rearrangement of the structure by atomic jumps over short distances. The existence of phasons was demonstrated by quasielastic MS for the first time by Coddens et al. [13].

${ }^{75} \mathrm{Fe}$ Mössbauer spectra of the icosahedral $\mathrm{Al}_{62} \mathrm{Cu}_{25.5} \mathrm{Fe}_{12.5}$ QC were measured in the temperature range $4.2-1090 \mathrm{~K}$ [14]. They are similar to the spectrum shown in Fig. 2. The temperature dependence of the resonant area derived from the spectra (Fig. 8) could be fitted well to the expression for a Debye-Waller factor within a Debye model of lattice vibrations for the data up to $580 \mathrm{~K}$. Above that temperature, the temperature dependence of the resonant area strongly deviates from the Debye model and no such fit is possible over the whole temperature 


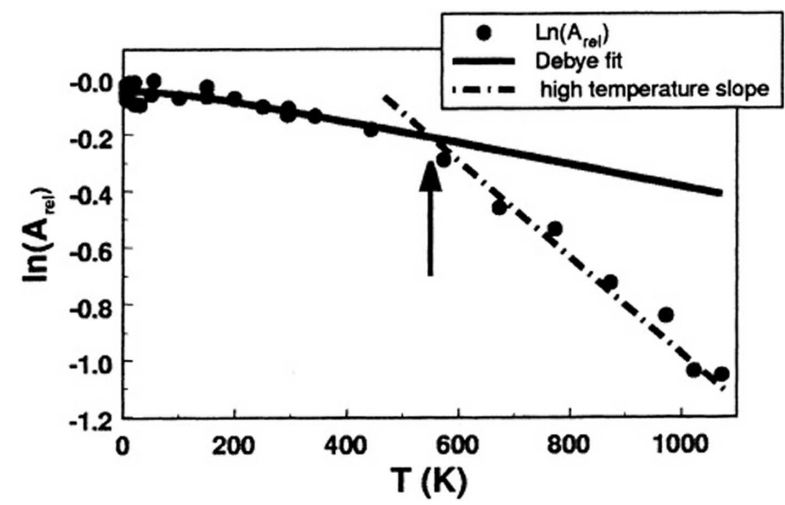

Fig. 8. The temperature dependence of the normalized resonant area for the icosahedral $\mathrm{Al}_{62} \mathrm{Cu}_{25.5} \mathrm{Fe}_{12.5}$. The solid line is a least-squares fit of the data below $580 \mathrm{~K}$ (marked by the arrow) to the expression for a Debye-Waller factor within a Debye model of lattice vibrations $[1$

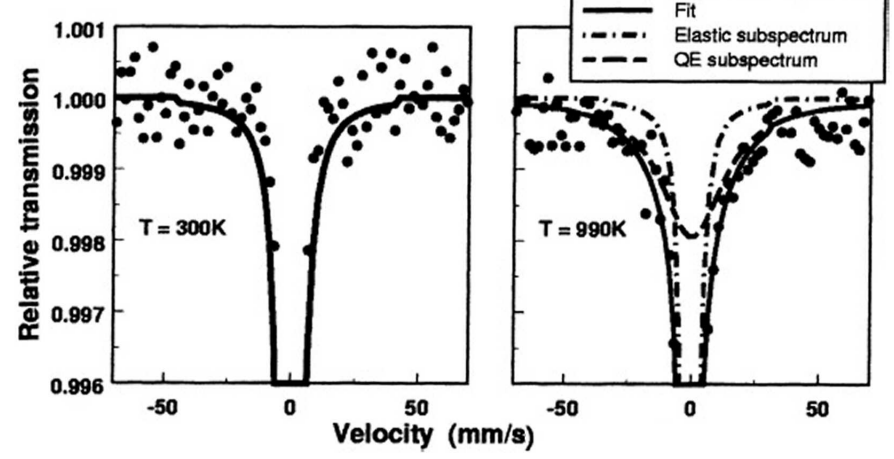

Fig. 9. High-velocity ${ }^{75} \mathrm{Fe}$ Mössbauer spectra of the icosahedral $\mathrm{Al}_{62} \mathrm{Cu}_{25.5} \mathrm{Fe}_{12.5}$ enriched to $100 \%$ with an ${ }^{75} \mathrm{Fe}$ isotope at 300 and $990 \mathrm{~K}$. The QE contribution to the $990 \mathrm{~K}$ spectrum is evident [14].

range. The change in the slope of the temperature dependence of the resonant area above $580 \mathrm{~K}$ (Fig. 8) indicates that the process of the phason-induced iron atomic jumps sets in. The missing area for temperatures above $580 \mathrm{~K}$ results from a new quasielastic (QE) component appearing at these temperatures which is distributed at large Doppler velocities. In order to detect this $\mathrm{QE}$ component, ${ }^{75} \mathrm{Fe}$ Mössbauer spectra of the icosahedral $\mathrm{Al}_{62} \mathrm{Cu}_{25.5} \mathrm{Fe}_{12.5}$ QC enriched to $100 \%$ with an ${ }^{75} \mathrm{Fe}$ isotope were measured at large Doppler velocities (Fig. 9). The Mössbauer spectrum at $300 \mathrm{~K}$ consists only of an elastic component, similar to the component observed at low Doppler velocities (Fig. 2). However, the Mössbauer spectrum at $990 \mathrm{~K}$ contains a broad QE component resulting from the phason-induced iron atomic jumps. The timescale of these atomic jumps, as calculated from the line width of the QE component, is about $3.3 \mathrm{~ns}$. 


\section{Acknowledgments}

This work was supported by the Natural Sciences and Engineering Research Council of Canada.

\section{References}

[1] D. Shechtman, I. Blech, D. Gratis, J.W. Cahn, Phys. Rev. Lett. 53, 1951 (1987).

[2] Physical Properties of Quasicrystals, Ed. Z.M. Stadnik, Springer-Verlag, Berlin 1999.

[3] Z.M. Stadnik, in: Mössbauer Spectroscopy Applied to Magnetism and Materials Science, Vol. 2, Eds. G.J. Long, F. Grandjean, Plenum Press, New York 1996, p. 125 .

[4] H. Takakura, C.P. Gómez, A. Yamamoto, M. De Boissieu, A.P. Tsai, Nature Mater. 6, 58 (2007).

[5] E. Cockayne, R. Phillips, X.B. Kan, S.C. Moss, J.L. Robertson, T. Ishimasa, M. Mori, J. Non-Cryst. Solids 153-154, 140 (1993).

[6] E.S. Zijlstra, J. Kortus, M. Krajčí, Z.M. Stadnik, S.K. Bose, Phys. Rev. B 69, 094206 (2004).

[7] Z.M. Stadnik, P. Wang, J. Phys. Condens. Matter 16, 7303 (2004).

[8] P.J. Steinhardt, H.-C. Jeong, K. Saitoh, M. Tanaka, E. Abe, A.P. Tsai, Nature 396, 55 (1998); Y. Yan, S.J. Pennycook, Phys. Rev. B 61, 14291 (2000); M. Mihalkovič, I. Al-Lehyani, E. Cockayne, C.L. Henley, N. Moghadam, J.A. Moriarty, Y. Wang, M. Widom, Phys. Rev. B 65, 104205 (2002); A. Cervellino, T. Haibach, W. Steurer, Acta Crystallogr. B 58, 8 (2002).

[9] B. Charrier, B. Ouladdiaf, D. Schmitt, Phys. Rev. Lett. 78, 4637 (1997).

[10] T.J. Sato, H. Takakura, A.P. Tsai, K. Shibata, Phys. Rev. Lett. 81, 2364 (1998); D.R. Noakes, G.M. Kalvius, R. Wäppling, C.E. Stronach, M.F. White Jr, H. Saito, K. Fukamichi, Phys. Lett. A 238, 197 (1998); Z. Islam, I.R. Fisher, J. Zarestky, P.C. Canfield, C. Stassis, A.I. Goldman, Phys. Rev. B 57, R11047 (1998).

[11] S. Iwano, H. Nishimoto, R. Tamura, S. Takeuchi, Philos. Mag. 86, 435 (2006).

[12] Z.M. Stadnik, K. Al-Qadi, P. Wang, J. Phys., Condens. Matter 19, 326208 (2007).

[13] G. Coddens, S. Lyonnard, B. Sepiol, Y. Calvayrac, J. Phys. I (France) 5, 771 (1995).

[14] R.A. Brand, J. Voss, Y. Calvayrac, Mater. Sci. Eng. A 294-296, 666 (2000). 